\title{
Levels of oxidative stress biomarkers in seminal plasma and their relationship with seminal parameters Ali Khosrowbeygi ${ }^{\dagger 1}$ and Nosratollah Zarghami* ${ }^{* 2}$
}

Address: ${ }^{1}$ Department of Biochemistry, School of Medicine, Lorestan University of Medical Sciences, Khoramabad, Iran and ${ }^{2}$ Department of Radio Pharmacy, Drug Applied Research Center, Tabriz University of Medical Sciences, Tabriz, Iran

Email: Ali Khosrowbeygi - khosrowbeygi@yahoo.com; Nosratollah Zarghami* - zarghami@tbzmed.ac.ir

* Corresponding author †Equal contributors

Published: I June 2007

BMC Clinical Pathology 2007, 7:6 doi:10.1 186/1472-6890-7-6

Received: 9 October 2006

Accepted: I June 2007

This article is available from: http://www.biomedcentral.com//472-6890/7/6

(C) 2007 Khosrowbeygi and Zarghami; licensee BioMed Central Ltd.

This is an Open Access article distributed under the terms of the Creative Commons Attribution License (http://creativecommons.org/licenses/by/2.0), which permits unrestricted use, distribution, and reproduction in any medium, provided the original work is properly cited.

\begin{abstract}
Background: There is growing evidence that damage to spermatozoa by reactive oxygen species (ROS) play a key role in male infertility. The aim of the present study was to assess seminal plasma levels of total antioxidant capacity (TAC), free 8-Isoprostane and activities of catalase and superoxide dismutase (SOD) in men with asthenozoospermia, asthenoteratozoospermia and oligoasthenoteratozoospermia compared with normozoospermic males.
\end{abstract}

Methods: The patients consisted of 46 men with seminal parameters abnormalities. The patients were grouped into asthenozoospermic $(n=15)$, asthenoteratozoospermic $(n=16)$ and oligoasthenoteratozoospermic $(n=15)$. The control group consisted of 16 healthy males with normozoospermia. Catalase activity was measured by Aebi spectrophotometeric method. Levels of TAC and SOD were measured by commercially available colorimetric assays. Level of free 8Isoprostane was assessed by commercially available enzyme immunoassay (EIA) method. Differences between groups were assessed using Mann-Whitney $U$ test and Kruskal-Wallis test. Coefficients of correlation were calculated using Spearman's correlation analysis. All hypothesis tests were two-tailed with statistical significance assessed at the $p$ value $<0.05$ level with $95 \%$ confidence intervals

Results: Levels of catalase and TAC were significantly lower in patients than the control group. No significant changes were seen in SOD activities. Levels of free 8-Isoprostane were significantly higher in patients than the control group. Furthermore, asthenozoospermic, asthenoteratozoospermic and oligoasthenoteratozoospermic groups had significantly lower values of catalase activity and TAC when compared to normozoospermic males. Levels of free 8Isoprostane were significantly higher in all patients subgroups than the control group. Levels of catalase and TAC were positively correlated with sperm motility and morphology. Free 8Isoprostane levels showed an inverse correlation with sperm motility and morphology.

Conclusion: Decreasing seminal plasma antioxidants levels, especially catalase and TAC, could have significant role in etiology of impaired sperm function. Measurement of 8-Isoprostane may be used as a specific biomarker for assessing oxidative stress on sperm. 


\section{Background}

In the etiology of male infertility, there is growing evidence that damage to spermatozoa by reactive oxygen species (ROS) play a key role $[1,2]$. Spermatozoa contain large quantities of polyunsaturated fatty acids (PUFA). Therefore, they are susceptible to ROS-induced damage. It has been suggested that ROS induce membrane lipid peroxidation in sperm [3-5]. The seminal plasma is well endowed with an array of antioxidants that act as free radical scavengers to protect spermatozoa against oxidative stress. Seminal plasma contains a number of enzymatic antioxidants such as superoxide dismutase (SOD) and catalase. In addition, it contains a variety of non-enzymatic antioxidants [6-9].

The findings on the seminal plasma catalase and SOD activities and total antioxidant capacity (TAC) are controversial. Sanocka et al study showed statistically significant change in activity of SOD in infertile men compared to normozoospermic samples. They also observed that the SOD activity exceeds values obtained for normozoospermic samples only in oligozoospermic males [10]. In another study Sanocka et al investigated activities of SOD and catalase in men with asthenozoospermia, teratozoospermia and oligozoospermia compared to normozoospermic males. Their study showed a significant elevation in intracellular activity of SOD and decreasing in catalase activity in infertile samples [11]. Zini et al study showed that seminal plasma activity of SOD in infertile men is significantly grater than in fertile men while catalase activity is not different between these groups [12]. The study conducted by Siciliano et al showed seminal plasma enzymatic (catalase and SOD) and nonenzymatic (TAC) antioxidant capacities do not alter in the asthenozoospermic specimens, whereas SOD activity is lower in oligoasthenozoospermic samples than normozoospermic males [13]. Hsieh et al investigation showed that there is not a significant difference in seminal plasma or sperm SOD activity between normozoospermic and oligo- or asthenozoospermic males [14]. This group also observed that activities of SOD do not correlate significantly with sperm motility and concentration. Tkaczuk-Wlach et al observed that whole semen SOD activity is higher in men with oligoszoospermia than those with normozoospermia [15]. Koca et al study showed that seminal plasma TAC in infertile asthenozoospermic and asthenoteratozoospermic males is lower than fertile men [16]. They also observed a positive correlation between seminal plasma TAC and sperm motility.

Available data on the impact of oxidative stress on sperm are based on the measurement of seminal plasma and sperm levels of malondialdehyde (MDA) by the thiobarbituric acid-reacting substance (TBARS) assay [17-25]. Recently, it has been shown that 8-Isoprostane is a spe- cific, chemically stable, and quantitative marker of oxidative stress in vivo. 8-Isoprostane is formed in situ in cell membranes; following free radical attack on the arachidonic acid [26-28].

The aim of the present study was to assess seminal plasma levels of TAC and free 8-Isoprostane and activities of catalase and SOD in men with asthenozoospermia, asthenoteratozoospermia and oligoasthenoteratozoospermia compared to normozoospermic males.

\section{Methods \\ Semen Samples}

A case-control study was designed. Following Institutional Review Board approval, the semen samples were collected from the case and the control groups. All specimens were collected into sterile plastic containers by masturbation after an abstinence period of 3-5 days, and were analyzed within $1 \mathrm{~h}$ of collection. After allowing at least $30 \mathrm{~min}$ for liquefaction to occur, semen analysis was performed to measure sperm concentration, sperm motility and sperm morphology using Sperm Quality Analyzer IIC (SQA IIC, United Medical Systems Inc, Santa Ana, CA, USA) $[29,30]$. Samples with a leukocyte concentration $>10^{6} / \mathrm{ml}$ of ejaculate and specimens with hyperviscosity were excluded from this study. The criteria for sperm normality were as follows: sperm concentration $\geq 20 \times 10^{6} /$ $\mathrm{ml}$ of ejaculate, sperm motility $\geq 50 \%$ and normal sperm morphology $\geq 30 \%[13,29,30]$. The case group consisted of men with asthenozoospermia $(\mathrm{n}=15)$ (age $31.33 \pm$ $4.84 \mathrm{yr}$ ), asthenoteratozoospermia $(\mathrm{n}=16)$ (age $34.31 \pm$ $5.20 \mathrm{yr}$ ) and oligoasthenoteratozoospermia $(\mathrm{n}=15)$ (age $35.75 \pm 5.33 \mathrm{yr}$ ). The control group consisted of 16 men with normal semen parameters and proven fertility (age $32.06 \pm 3.91 \mathrm{yr}$ ). Liquefied semen samples were centrifuged at $10000 \mathrm{~g}$ for 10 minutes [12,31]. The supernatant seminal plasma was then frozen at $-80^{\circ} \mathrm{C}$ until examination.

\section{TAC measurement}

TAC was measured by colorimetric assay $[32,33]$. We used commercially available colorimetric method (Randox Laboratories Ltd, UK). The frozen seminal plasma was thawed by placing the vials in a water bath at $37^{\circ} \mathrm{C}$ for 20 minutes and immediately assessed for its antioxidant capacity. Twenty microliters of seminal plasma was added to $1 \mathrm{~mL}$ of the reconstituted chromogen, 2, 2'-Azino-di(3-ethylbenzthiazoline sulphonate) (ABTS)-metmyoglobin $(10 \mathrm{~mL}$ vial with $10 \mathrm{~mL}$ of phosphate-buffered saline buffer). Twenty microliters of Trolox (6-hydroxyl-2, 5, 7, 8-tetramethylchroman-2-carboxylic acid) at a concentration of $1.71 \mathrm{mmol} / \mathrm{L}$ was used as the standard. Whereas $20 \mu \mathrm{l}$ of deionized water was used as a blank. One milliliter of chromogen was added to the standard and blank samples. With spectrophotometer adjusted at a 
wavelength of $600 \mathrm{~nm}$, the initial absorbance (A1) was read. Two hundred microliters of $\mathrm{H}_{2} \mathrm{O}_{2}(250 \mu \mathrm{mol} / \mathrm{L})$ was then added to all tubes, and absorbance (A2) was read exactly after 3 minutes. The difference between $\mathrm{A} 2$ and $\mathrm{A} 1$ $(\triangle \mathrm{A})$ was calculated. The TAC of the sample was then calculated by the following formula: $\mathrm{TAC}=$ Concentration of the Standard $\times(\Delta \mathrm{A}$ Blank $-\Delta \mathrm{A}$ Sample $) /(\Delta \mathrm{A}$ Blank $-\Delta \mathrm{A}$ Standard). The results were expressed as $\mathrm{mM}$.

\section{SOD activity measurement}

SOD activity was measured by colorimetric assay $[12,31]$. We used commercially available colorimetric method (Randox Laboratories Ltd, UK). This method employs xanthine and xanthine oxidase to generate superoxide radicals which reacts with 2-(4-iodophenyl)-3-(4-nitrophenol)-5-phenyltetrazoliumchloride (I.N.T) to form red formazan dye. The SOD activity is then measured by the degree of inhibition of this reaction. One unit of SOD inhibits reduction of INT by $50 \%$ under the conditions of the assay. After thawing, the seminal plasma was diluted 30 -fold with $10 \mathrm{mM}$ phosphate buffer, $\mathrm{pH}$ 7.0. Assay was performed at $37^{\circ} \mathrm{C}$. Phosphate buffer was used as blank. Mixed substrate and xanthine oxidase were added into standards and sample tubes and vortexed well. With spectrophotometer adjusted at a wavelength of $505 \mathrm{~nm}$, the initial absorbance (A1) was read. Final absorbance (A2) was read exactly after 3 minutes. Percentages of inhibition of standards and samples were calculated. The SOD activity was measured using calibration curve of percentage inhibition for each standard against $\log _{10}$ of standards and SOD activity was expressed as $\mathrm{U} / \mathrm{ml}$.

\section{Catalase activity measurement}

Catalase activity was estimated by the method of Aebi [34]. Catalase can degrade hydrogen peroxide which can be measured directly by the decrease in the absorbance at $240 \mathrm{~nm}$. The hydrogen peroxide was diluted with phosphate buffer $\mathrm{pH} 7.0$ and its initial absorbance was adjusted between 0.5 to 0.6 absorbance unit at $240 \mathrm{~nm}$. The decrease in the absorbance was measured. One unit of catalase activity was defined as the amount of catalase which absorbed in $30 \mathrm{sec}$ at $25^{\circ} \mathrm{C}$. The catalase activity was then calculated from the change in absorbance and finally expressed as $\mathrm{U} / \mathrm{ml}$.

\section{8-Isoprostane assessment}

We assessed free form of 8-Isoprostane and only the fraction shedded to seminal plasma from cell membranes.

\section{Free 8-Isoprostane purification}

Free 8-Isoprostane was purified by affinity chromatography method [35]. We used commercially available affinity column (Cayman Chemical, Ann Arbor, MI, USA). All samples were centrifuged at $15000 \mathrm{~g}$ for isolating of particulates and precipitates. Then the supernatant was diluted 1:5 with column buffer and applied to the column. Other procedures were according to the instructions provided by the manufacturer. The ethanol washed 8-Isoprostane stored at $-80^{\circ} \mathrm{C}$ until measurement.

\section{Free 8-Isoprostane measurement}

At first, the elution solution was evaporated to dryness using a vacuum centrifugation. Then, the concentration of free 8-Isoprostane was measured by enzyme immunoassay (EIA) method [35]. We used commercially available EIA method (Cayman Chemical, Ann Arbor, MI, USA). The procedure for the EIA was according to the instructions provided by the manufacturer. The sample volume that used was $50 \mu \mathrm{l}$. Absorbance was measured at a wavelength of $405 \mathrm{~nm}$ using enzyme-linked immunosorbent assay (ELISA) reader (STAT FAX 2100, USA). The levels of free 8-Isoprostane were presented as $\mathrm{ng} / \mathrm{ml}$. The intra-assay coefficient of variation was $<10 \%$.

\section{Statistical analysis}

Differences between groups were assessed using MannWhitney U test and Kruskal-Wallis test. Coefficients of correlation were calculated using Spearman's correlation analysis. All hypothesis tests were two-tailed with statistical significance assessed at the $\mathrm{p}$ value $<0.05$ level with $95 \%$ confidence intervals. The data were expressed as the mean \pm SEM. Statistical computations were calculated using SPSS 11.5 for windows software (SPSS Inc, Chicago, IL, USA).

\section{Results}

Seminal parameters of the subjects are reported in Table 1. Table 2 shows comparison of seminal plasma levels of free 8-Isoprostane and TAC and activities of catalase and SOD between patients and control groups. Levels of catalase and TAC were significantly lower in patients than the control group. No significant changes were seen in SOD activities. Seminal plasma levels of free 8-Isoprostane were significantly higher in patients than the control group. Table 3 shows seminal plasma levels of free 8-Isoprostane and TAC and activities of catalase and SOD in subgroups of the patients compared to normozoospermic subjects. Asthenozoospermic, asthenoteratozoospermic and oligoasthenoteratozoospermic males had significant lower values of catalase and TAC compared with normozoospermic males. Levels of free 8-Isoprostane were significantly higher in all patients than the control group.

Then, we examined the correlation between seminal parameters and seminal plasma levels of 8-Isoprostane, TAC, SOD and catalase in total case group. Levels of TAC showed a positive correlation with sperm motility $(\mathrm{r}=$ $0.45, \mathrm{p}<0.05)$ and morphology $(\mathrm{r}=0.45, \mathrm{p}<0.05)$. We also observed a direct correlation between catalase activity and sperm concentration $(\mathrm{r}=0.41, \mathrm{p}<0.05)$, sperm 
Table I: Seminal parameters in controls and subgroups of patients.

\begin{tabular}{lccc}
\hline Diagnosis & Concentration $(106 / \mathrm{ml})$ & Motility (\%) & Morphology $(\%)$ \\
\hline Controls & & & \\
$\quad$ Normozoospermic $(n=16)$ & $97.06 \pm 5.38$ & $57.81 \pm 1.39$ & $39.62 \pm 1.04$ \\
Patients & & & \\
$\quad$ Asthenozoospermic $(n=15)$ & $62.40 \pm 1.90$ & $46.07 \pm 0.30$ & $31.07 \pm 0.25$ \\
$\quad$ Asthenoteratozoospermic $(n=16)$ & $39.00 \pm 2.75$ & $36.75 \pm 1.38$ & $23.56 \pm 0.89$ \\
$\quad$ Oligoasthenoteratozoospermic $(n=15)$ & $13.73 \pm 1.19$ & $18.53 \pm 1.28$ & $16.80 \pm 0.33$ \\
\hline
\end{tabular}

Data are reported as mean \pm SEM.

motility $(\mathrm{r}=0.41, \mathrm{p}<0.05)$, and sperm morphology $(\mathrm{r}=$ 0.42 , p < 0.05). Seminal plasma levels of free 8-Isoprostane showed an inverse correlation with sperm motility ( $\mathrm{r}$ $=-0.26, \mathrm{p}<0.05)$ and sperm morphology $(\mathrm{r}=-0.27, \mathrm{p}<$ $0.05)$. Levels of SOD did not show any correlation with seminal parameters.

\section{Discussion}

The most relevant findings of this study were (i) significant elevation of seminal plasma levels of free 8-Isoprostane and decreasing of catalase and TAC levels in asthenozoospermic, asthenoteratozoospermic, oligoasthenoteratozoospermic samples compared with normozoospermic men (ii) both catalase and TAC showed a positive correlation with sperm motility and morphology while an inverse correlation was seen with free 8-Isoprostane levels.

Our findings about activities of SOD were contradicted the results of Sanocka et al $[10,11]$. Our findings confirm Sanocka et al results about catalase activity in infertile men [11]. Siciliano et al. evaluated antioxidant capacity of seminal plasma in asthenozoospermic and oligoasthenozoospermic specimens with normal viscosity and hyperviscosity [13]. Their study showed that in semen with normal viscosity levels of catalase, SOD and TAC do not alter in asthenozoospermic specimens compared with normozoospermic men. They observed that SOD activity declines in oligoasthenozoospermic males. In contrast to Siciliano et al., we observed a significant decrease in cata-

Table 2: Seminal plasma levels of free 8-Isoprostane and total antioxidant capacity (TAC) and activities of catalase and superoxide dismutase (SOD) in controls and total patients.

\begin{tabular}{lcc}
\hline Variables & Controls $(\mathrm{N}=16)$ & Patients $(\mathrm{N}=46)$ \\
\hline TAC $(\mathrm{mM})$ & $1.63 \pm 0.08$ & $1.05 \pm 0.04^{\mathrm{a}}$ \\
Catalase $(\mathrm{U} / \mathrm{ml})$ & $22.58 \pm 2.20$ & $14.40 \pm 0.93^{\mathrm{b}}$ \\
SOD $(\mathrm{U} / \mathrm{ml})$ & $5.89 \pm 0.96$ & $5.32 \pm 0.56$ \\
8-lsoprostane $(\mathrm{ng} / \mathrm{ml})$ & $2.60 \pm 0.38$ & $18.23 \pm 3.56^{\mathrm{c}}$ \\
\hline
\end{tabular}

Data are reported as mean \pm SEM.

${ }^{a} p=0.001,{ }^{b} p=0.0 .03,{ }^{c} p=0.0001$ in comparison with controls. lase activity and TAC in men with asthenozoospermia compared to normozoospermic men. Our finding about SOD activity in asthenozoospermic men was similar to Siciliano et al study. In another study, Hsieh et al evaluated SOD activities in seminal plasma and spermatozoa in infertile men with normozoospermia and oligoasthenozoospermia [14]. They observed that SOD activities of seminal plasma and sperm in both groups are nonsignificantly different. Hsieh et al also observed that SOD activities of seminal plasma and sperm are positively but nonsignificantly correlated with sperm motility and concentration. Our results about the SOD activity of the seminal plasma in oligoasthenozoospermic men were similar to Hsieh et al study. Koca et al evaluated TAC in infertile asthenozoospermic and asthenoteratozoospermic men compared to normozoosperic fertile men [16]. Their study showed that asthenozoospermic and asthenoteratozoospermic males have significantly lower mean TAC value than the control group. Koca et al study also showed that TAC correlates positively to sperm motility. We also observed these findings. Tkaczuk-Wlach et al evaluated activity of SOD in the whole semen of patients with oligozoospermia compared to patients with normozoospermia [15]. Their study showed that oligozoospermia males have significantly higher mean SOD activity than the control group. However, the finding of Tkaczuk-Wlach et al study was limited by the fact that they used whole semen sample, because the membrane-bound oxidases or antioxidants associated with cellular debris and/or organelles can influence activities of antioxidant enzymes such as SOD and catalase [12].

Immature spermatozoa with abnormal morphology and cytoplasmic retention are the most sources of ROS production in semen. This has been confirmed by Gil-Guzman et al study [36]. Their study showed that there is a direct significant correlation between ROS levels and the rate of abnormal forms in semen. Gil-Guzman et al also observed that there is an inverse significant correlation between seminal plasma TAC and ROS levels. They suggested that the inverse correlation between TAC and ROS might be associated with an increase in the consumption 
Table 3: Seminal plasma levels of free 8-Isoprostane and total antioxidant capacity (TAC) and activities of catalase and superoxide dismutase (SOD) in controls and subgroups of patients.

\begin{tabular}{|c|c|c|c|c|}
\hline Diagnosis & 8-Isoprostane $(\mathrm{ng} / \mathrm{ml})$ & TAC (mM) & Catalase $(\mathrm{U} / \mathrm{ml})$ & $\mathrm{SOD}(\mathrm{U} / \mathrm{ml})$ \\
\hline \multicolumn{5}{|l|}{ Controls } \\
\hline Normozoospermic $(n=16)$ & $6.95 \pm 2.10$ & $1.63 \pm 0.08$ & $22.58 \pm 2.20$ & $5.89 \pm 0.96$ \\
\hline \multicolumn{5}{|l|}{ Patients } \\
\hline Asthenozoospermic $(n=15)$ & $14.66 \pm 4.10^{\mathrm{a}}$ & $1.12 \pm 0.06 \mathrm{~d}$ & $13.76 \pm 1.64 \mathrm{e}$ & $4.82 \pm 0.54$ \\
\hline Asthenoteratozoospermic $(n=16)$ & $16.7 \mid \pm 5.58^{b}$ & $1.02 \pm 0.08$ & $16.66 \pm 1.46^{f}$ & $6.24 \pm 1.50$ \\
\hline Oligoasthenoteratozoospermic $(n=15)$ & $23.42 \pm 8.36^{c}$ & $1.01 \pm 0.09 \mathrm{~d}$ & $12.61 \pm 1.658$ & $4.82 \pm 0.39$ \\
\hline
\end{tabular}

Data are reported as mean \pm SEM.

${ }^{a} p=0.01,{ }^{b} p=0.008,{ }^{c} p=0.04,{ }^{d} p=0.0001,{ }^{e} p=0.004,{ }^{f} p=0.04,{ }^{g} p=0.001$ in comparison with normozoospermic men.

of soluble, non-enzymatic antioxidants in seminal plasma which is resulted from over production of ROS. In our study the correlation between TAC and sperm morphology was positive. According to Gil-Guzman et al study, this finding could be interpreted that in semen with high rate of abnormal forms, because of high levels of ROS production, consumption of non-enzymatic antioxidants will be higher.

The inverse correlation between lipid peroxidation and sperm motility has been shown by Keskes-Ammar et al [24]. In our study both TAC and catalase, two defenses against ROS, showed direct correlation with sperm motility. Keskes-Ammar et al and our study might suggest that the higher level of antioxidant status prevents lipid peroxidation in spermatozoa and therefore results in higher sperm motility. Hsieh et al observed a slightly positive correlation between seminal plasma SOD activity and sperm concentration [14]. Their interpretation was that higher concentrations of spermatozoa might produce higher levels of SOD. The positive significant correlation between seminal plasma catalase activity and sperm concentration that observed in our study may be interpreted similar to Hsieh et al. Immature spermatozoa generate primary superoxide anion. This anion is dismuted to hydrogen peroxide by SOD activity. Detoxification of hydrogen peroxide is carried out by catalase activity. Hydrogen peroxide is the primary toxic ROS for human spermatozoa that its high concentration induces lipid peroxidation and results in cell death. Therefore, the balance of the SOD and catalase activities in semen is important for maintaining sperm motility [14].

Our results are agreed with some previous studies that show increasing of lipid peroxidation by measuring MDA in sperm and seminal plasma in males with asthenozoospermia, asthenoteratozoospermia and oligoasthenoteratozoospemia $[20,23]$. Similar to MDA $[18,24] 8$ Isoprostane also showed an inverese correlation with sperm motility.
MDA is widely used index of lipid peroxidation due to its simplicity. The TBARS test application to body fluids and tissue samples is unreliable. Application of a gas chromatography/mass spectrometry (GC/MS) assay for MDA has indicated that the commonly used TBARS assay overestimates the actual MDA levels by more than 10-fold, possibly resulting from cross reactivity with other aldehydes and the harsh conditions used in sample preparation [26].

Recent studies have focused on 8-Isoprostane, as an index of lipid peroxidation. Isoprostanes are formed in situ in cell membranes; following free radical attack on the arachidonic acid. Unlike prostaglandins, which are formed from arachidonic acid following its release from the $s n-2$ position of phospholipids by phospholipase $\mathrm{A}_{2}$, isoprostanes are formed initially in situ, where they may contribute to the effects of oxidative stress on membrane biophysics. Measurement of 8-Isoprostane may provide a reliable marker of lipid peroxidation in vivo, because, it is a stable compound. In addition, 8-Isoprostane is specific product of free radical-induced lipid peroxidation. 8-Isoprostane has also been found to be present in detectable quantities in all normal biological tissues and in free form in all normal biological fluids. This is important because it allows the definition of a normal range such that small increases in its formation can be detected in situations of mild oxidant stress. Finally, the levels of 8-Isoprostane is unaffected by lipid content of the diet $[26,28]$.

Evidence is beginning to emerge suggesting that isoprostanes are not only markers of oxidative injury, but active participants in the pathophysiology of some disorders. The capacity of isoprostanes to readily esterify to cell lipid membranes, and the resulting marked distortion of membrane structure and function, undoubtedly contribute to their pathophysiologic potential. As well, the existence of specific receptor for isoprostanes has been proven [37]. So, because isoprostanes are biologically active, they may have significant role in the etiology of some sperm function abnormality. 


\section{Conclusion}

It is concluded that decreasing seminal plasma antioxidant status, especially catalase activity and TAC, may have significant role in the etiology of impaired sperm function. Measurement of 8-Isoprostane may be used as a specific biomarker for assessing oxidative stress on sperm. However, further studies with a larger sample size are required to confirm these findings.

\section{Competing interests}

The author(s) declare that they have no competing interests.

\section{Authors' contributions}

Ali Khosrowbeygi carried out all of the experiment and participated in data analyzing and writing the manuscript.

Nosratollah Zarghami designed the study and participated in data analyzing and writing the manuscript.

\section{Acknowledgements}

This research was granted by Drug Applied Research Center of Tabriz University of Medical Sciences.

\section{References}

I. Agarwal A, Saleh RA: Role of oxidants in male infertility: rationale, significance, and treatment. Urol Clin North Am 2002 29:817-827.

2. Agarwal A, Saleh RA, Bedaiwy MA: Role of reactive oxygen species in the pathophysiology of human reproduction. Fertil Steril 2003, 79:829-843.

3. Esfandiari N, Sharma RK, Saleh RA, Thomas AJ Jr, Agarwal A: Utility of the nitrobluetetrazolium reduction test for assessment of reactive oxygen species production by seminal leukocytes and spermatozoa. J Androl 2003, 24:862-870.

4. Agarwal A, Nallella KP, Allamaneni SS, Said TM: Role of antioxidants in treatment of male infertility: an overview of the literature. Reprod Biomed Online 2004, 8:616-627.

5. Sanocka D, Kurpisz M: Reactive oxygen species and sperm cells. Reprod Biol Endocrinol 2004, 2: 12

6. Agarwal A, Prabakaran SA: Mechanism, measurement, and prevention of oxidative stress in male reproductive physiology. Indian J Exp Biol 2005, 43:963-974.

7. Baker MA, Aitken RJ: Reactive oxygen species in spermatozoa: methods for monitoring and significance for the origins of genetic disease and infertility. Reprod Biol Endocrinol 2005, 3:67.

8. Agarwal A, Prabakaran S, Allamaneni SS: Relationship between oxidative stress, varicocele and infertility: a meta-analysis. Reprod Biomed Online 2006, 12:630-633.

9. Agarwal A, Gupta S, Sikka S: The role of free radicals and antioxidants in reproduction. Curr Opin Obstet Gynecol 2006, I 8:325-332.

10. Sanocka D, Miesel R, Jedrzejczak P, Kurpisz MK: Oxidative stress and male infertility. I Androl 1996, I 7:449-454.

II. Sanocka D, Miesel R, Jedrzejczak P, Chelmonska-Soyta AC, Kurpisz M: Effect of reactive oxygen species and the activity of antioxidant systems on human semen: association with male infertility. Int J Androl 1997, 20:255-264.

12. Zini A, Garrels K, Phang D: Antioxidant activity in the semen of fertile and infertile men. Urology 2000, 55:922-926.

13. Siciliano L, Tarantino P, Longobardi F, Rago V, De Stefano C, Carpino $A$ : Impaired seminal antioxidant capacity in human semen with hyperviscosity or oligoasthenozoospermia. J Androl 2001 , 22:798-803.

14. Hsieh YY, Sun YL, Chang CC, Lee YS, Tsai HD, Lin CS: Superoxide dismutase activities of spermatozoa and seminal plasma are not correlated with male infertility. J Clin Lab Anal 2002 16:127-|3|.

15. Tkaczuk-Wlach J, Kankofer M, Jakiel G: Activity of superoxide dismutase and glutathione peroxidase in human semen in nor mozoospermia and spermatopathy. Ann Univ Mariae Curie Sklodowska 2002, 57(2):369-375.
16. Koca Y, Ozdal OL, Celik M, Unal S, Balaban N: Antioxidant activity of seminal plasma in fertile and infertile men. Arch Androl 2003 49:355-359.

17. Suleiman SA, Ali ME, Zaki ZM, el-Malik EM, Nasr MA: Lipid peroxidation and human sperm motility: protective role of vitamin $E$. J Androl 1996, 1 7:530-537.

18. Gomez E, Irvine DS, Aitken RJ: Evaluation of a spectrophotometric assay for the measurement of malondialdehyde and 4hydroxyalkenals in human spermatozoa: relationships with semen quality and sperm function. Int J Androl 1998, 2 I:8I-94.

19. Rhemrev JP, Vermeiden JP, Haenen GR, De Bruijne JJ, Rekers-Mombarg LT, Bast A: Progressively motile human spermatozoa are well protected against in vitro lipid peroxidation imposed by induced oxidative stress. Andrologia 2001, 33:151-158.

20. Fraczek M, Szkutnik D, Sanocka D, Kurpisz M: Peroxidation components of sperm lipid membranes in male infertility. Ginekol $\mathrm{Po}$ 200I, 72:73-79.

21. Laudat A, Lecourbe k, Guechot J, Palluel AM: Values of sperm thiobarbituric acid-reactive substance in fertile men. Clin Chim Acta 2002, 325: I13-II5

22. Dandekar SP, Nadkarni GD, Kulkarni VS, Punekar S: Lipid peroxidation and antioxidant enzymes in male infertility. J Postgrad Med 2002, 48: 186-189.

23. Nakamura H, Kimura T, Nakajima A, Shimoya K, Takemura M, Hashimoto K, Isaka S, Azuma C, Koyama M, Murata Y: Detection of oxidative stress in seminal plasma and fractionated sperm from subfertile male patients. Eur J Obstet Gynecol Reprod Biol 2002, 105: $155-160$.

24. Keskes-Ammar L, Feki-Chakroun N, Rebai T, Sahnoun Z, Ghozzi H, Hammami S, Zghal K, Fki H, Damak J, Bahloul A: Sperm oxidative stress and the effect of an oral vitamin $E$ and selenium supplement on semen quality in infertile men. Arch Androl 2003 , 49:83-94.

25. Tavilani H, Doosti M, Saeidi H: Malondialdehyde levels in sperm and seminal plasma of asthenozoospermic and its relationship with semen parameters. Clin Chem Acta 2005, 356:199-203.

26. Meagher EA, FitzGerald GA: Indices of lipid peroxidation in vivo: strengths and limitations. Free Radic Biol Med 2000, 28: I745-I750.

27. Roberts LJ, Morrow JD: Measurement of $F(2)$-isoprostanes as an index of oxidative stress in vivo. Free Radic Biol Med 2000, 28:505-513.

28. Pratico D, Lawson JA, Rokach J, FitzGerald GA: The isoprostanes in biology and medicine. Trends Endocrinol Metab 200I, I 2:243-247.

29. Shibahara H, Suzuki T, Obara H, Hirano Y, Onagawa T, Taneichi A Takamizawa S, Sato I: Accuracy of the normal sperm morphology value by Sperm Quality Analyzer IIC: comparison with the strict criteria. Int J Androl 2002, 25:45-48

30. Suzuki T, Shibahara H, Tsunoda H, Hirano Y, Taneichi A, Obara H, Takamizawa S, Sato I: Comparison of the Sperm Quality Analyzer IIC variables with the computer-aided sperm analysis estimates. Int J Androl 2002, 25:49-54

31. Zini A, Fischer MA, Mak V, Phang D, Jarvi K: Catalase-like and superoxide dismutase-like activities in human seminal plasma. Urol Res 2002, 30:321-323.

32. Said TM, Kattal N, Sharma RK, Sikka SC, Thomas AJ Jr, Mascha E, Agarwal A: Enhanced chemiluminescence assay vs colorimetric assay for measurement of the total antioxidant capacity of human seminal plasma. J Androl 2003, 24:676-680.

33. Meucci E, Milardi D, Mordente A, Martorana GE, Giacchi E, De Marinis $L$, Mancini $A$ : Total antioxidant capacity in patients with varicoceles. Fertil Steril 2003:1577-1583.

34. Aebi H: Catalase in vitro. Methods Enzymol 1984, 105:121-126.

35. Nonaka-Sarukawa M, Yamamoto K, Aoki H, Takano H, Katsuki T, lkeda $U:$ Increased urinary I5-F2t-isoprostane concentrations in patients with non-ischaemic congestive heart failure: a marker of oxidative stress. Heart 2003, 89:87|-874.

36. Gil-Guzman E, Ollero M, Lopez MC, Sharma RK, Alvarez JG, Thomas A Jr, Agarwal A: Differential production of reactive oxygen species by subsets of human spermatozoa at different stages of maturation. Hum Reprod 2001, 16:1922-1930.

37. Habib A, Badr KF: Molecular pharmacology of isoprostanes in vascular smooth muscle. Chem Phys Lipids 2004, I 28:69-73.

\section{Pre-publication history}

The pre-publication history for this paper can be accessed here:

http://www.biomedcentral.com/1472-6890/7/6/prepub 